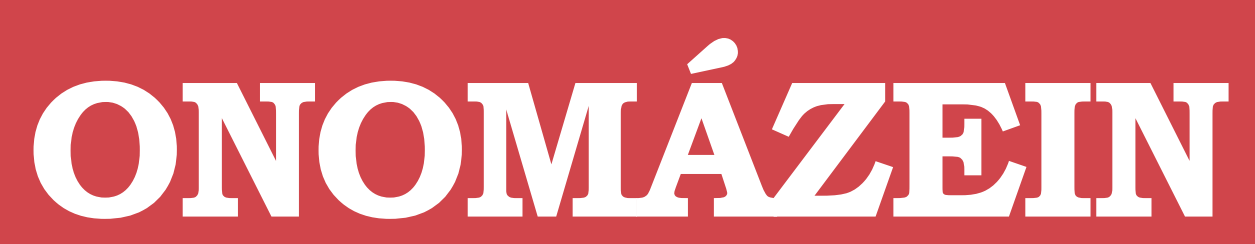

Revista de lingüística, filología y traducción
PONTIFICIA UNIVERSIDAD

\title{
Las jerarquías de argumentos nominales desde el enfoque de los proto-papeles temáticos ${ }^{1}$
}

Nominal argument hierarchies under the thematic proto-role approach

\author{
Álvaro Recio Diego \\ Universidad de Salamanca \\ España
}

\section{(c) $(i)$}

Álvaro Recio Diego: Departamento de Lengua Española, Facultad de Filología, Universidad de Salamanca, España. | Correo electrónico: alrecio@usal.es 


\section{Resumen}

El objeto de este trabajo es proponer un principio de proyección de argumentos en español capaz de explicar la estructura argumental en todos los sintagmas nominales (SSNN) con red temática, ya sea esta heredada (sustantivos deverbales y deadjetivales) o inherente (sustantivos relacionales y de representación). El enfoque de los proto-papeles temáticos, asumido tanto para verbos como para sustantivos, y la prueba de la posesivización, que determina cuál es el argumento más prominente, sugieren la existencia de dos jerarquías temáticas diferentes para la ordenación de los complementos del nombre: \{Poseedor > Proto-Agente > Proto-Paciente\}, para los SSNN relacionados con verbos; \{Proto-Todo > Proto-Parte\}, para los SSNN no relacionados con verbos.

Palabras clave: estructura argumental; sintagma nominal; jerarquías temáticas; proto-papeles temáticos; posesivización.

\section{Abstract}

The aim of this research is to propose a principle of argument projection which accounts for argument structure in every type of Noun Phrase with a thematic network, independently of this being inherited (deverbal and deadjectival nouns) or inherent (relational and 'picture' nouns). The thematic proto-role approach, assumed both for verbs and for nouns, and the possessivization test, which determines which of the arguments is the most prominent, suggest the existence of two different hierarchies for the ordering of noun complements: \{Possessor > Proto-Agent > Proto-Patient\} for verb-related Noun Phrases and \{Proto-Whole > Proto-Part\} for those non verb-related.

Keywords: argument structure; noun phrase; thematic hierarchies; thematic proto-roles; possessivization.

1 Este trabajo se ha beneficiado de la ayuda AP2007-00244 del programa FPU del Ministerio de Ciencia e Innovación de España. Agradezco a Julio Borrego, Ignacio Bosque, Luis López, Kay González y David Adger por sus comentarios, críticas y sugerencias, así como a los asistentes al XXXIX Romance Linguistics Seminar de la University of Cambridge (Reino Unido) y al Linguistics Circle de Wake Forest University (EEUU). Los errores que puedan permanecer son exclusivamente responsabilidad mía. 


\section{Introducción}

La mayor parte de los estudios sobre la organización interna y la estructura argumental del sintagma nominal (SN) parten del paralelismo con la oración y el entorno verbal (Chomsky, 1970; Cinque, 1980; Demonte, 1985; Abney, 1987; Grimshaw, 1990; Giorgi y Longobardi, 1991; Escandell, 1995; Siloni, 1997; Picallo, 1999; Longobardi, 2003; Ticio, 2005; Alexiadou y otros, 2007; Giusti, 2008; Ticio, 2010; Reuland, 2011; Peris, 2012; Roca, 2012; Fábregas, 2014). Por este motivo, suelen centrarse casi exclusivamente en dos clases de sustantivos: Ios deverbales (destrucción, interpretación), que heredan su estructura argumental de los verbos y mantienen así papeles temáticos como agente o tema, y los de representación o icónicos (foto, cuadro), vinculados por su naturaleza semántica a un predicado verbal. Sin embargo, existen otras clases de sustantivos que también seleccionan argumentos pero no se ajustan a los mismos patrones verbales de organización argumental: los deadjetivales (rapidez, sinceridad), con argumentos heredados de adjetivos, y los relacionales (madre, amigo), con red temática inherente debido a sus propiedades léxico-semánticas.

El análisis del funcionamiento de una serie de sintagmas nominales en español sugiere que, con independencia de si tal red es heredada en unos o intrínseca en otros, los nombres deverbales y de representación conforman un bloque común porque su estructura argumental es de naturaleza verbal y se ajusta a parámetros similares a los oracionales, mientras que los deadjetivales se agrupan con los relacionales en otro bloque porque su estructura argumental es de naturaleza estrictamente nominal y se ajusta a unas pautas de proyección de argumentos diferentes a las oracionales².

\section{Fundamentos teóricos}

Este trabajo asume una serie de principios relacionados con la estructura argumental de los predicados, la existencia de unos prototipos de papeles temáticos, el paralelismo parcial entre las estructuras nominales y verbales, así como la organización jerárquica de los complementos del nombre y su comprobación empírica a través de la prueba de la posesivización.

\subsection{Teoría temática y estructura argumental}

La teoría temática y la estructura argumental (Grimshaw, 1990) establece que los predicados seleccionan una red de papeles temáticos o papeles- $\theta$ (agente, tema, experimentante, receptor,

2 Para una revisión teórica exhaustiva de los tipos de sustantivos que seleccionan argumentos, así como de los paralelismos y las diferencias entre la estructura argumental nominal y la verbal, puede consultarse Recio (2015: 79-119). 
beneficiario...) que se proyectan en forma de argumentos sintácticos (sujeto, objeto...). El criterio temático o criterio- $\theta$ (Chomsky, 1981: 36) establece una correspondencia biunívoca entre papeles temáticos y argumentos sintácticos. A su vez, la hipótesis de la uniformidad de la asignación temática o HUAT (Baker, 1988: 46) formula la existencia de una correlación entre la representación semántica y la representación estructural de los argumentos, de modo que las relaciones temáticas entre argumentos han de tener reflejo también en la configuración sintáctica.

Los papeles- $\theta$ se organizan en una jerarquía de prominencia abstracta (Jackendoff, 1972), de tal manera que la realización de los argumentos sintácticos se basa en la posición de cada papel en dicha jerarquía. El papel más prominente se asigna, pues, al argumento más relevante en la estructura sintáctica, el sujeto; el segundo papel más prominente en el plano semántico se asigna al segundo papel más relevante en el plano sintáctico, etc. Son numerosas las jerarquías temáticas que se han propuesto en las últimas décadas; a continuación se señalan algunas de ellas:

(1) a. Agente > Meta/Fuente/Locativo > Tema (Jackendoff, 1972: 43)

b. Agente > Beneficiario > Receptor/Experimentante $>$ Instrumento $>$ Tema / Paciente $>$ Locativo

(Bresnan y Kanerva, 1989: 23)

c. Agente > Experimentante > Meta/Fuente/Locativo > Tema (Grimshaw, 1990: 8)

d. Agente > Experimentante > Tema > Locativo > Manera / Tiempo > Razón (Gutiérrez Bravo, 2007: 270)

Sin embargo, los papeles- $\theta$ y las jerarquías temáticas no están exentos de dificultades de análisis (Pesetsky, 1995: 11-54; Bosque y Gutiérrez-Rexach, 2009: 276-282). Como puede observarse, el consenso sobre el orden específico de los papeles- $\theta$ prácticamente queda reducido a la consideración del agente como el papel prominente. Las divergentes propuestas de (1) constatan tal desacuerdo: a pesar de que en todas ellas el agente ocupa el primer lugar, es clara la discrepancia en cuanto al resto de papeles- $\theta$, tanto en cuáles de ellos deben incluirse en la jerarquía como en su posición exacta.

\subsection{El enfoque de los proto-papeles temáticos}

La teoría de los proto-papeles temáticos (Dowty, 1991) reduce la multiplicidad de papeles- $\theta$ a dos prototipos conceptuales: el Proto-Agente y el Proto-Paciente. Cada uno de ellos reúne una serie de propiedades o implicaciones léxicas que operan en conjunto, sin que ninguna de ellas sea por sí sola necesaria o suficiente (Dowty, 1991: 572): 
(2) Proto-Agente
a. implicación volitiva en el evento o estado
b. conciencia y/o percepción
c. causación de un evento o cambio de estado en otro participante
d. movimiento (en relación con la posición de otro participante)
e. existencia independiente del evento designado por el verbo

(3) Proto-Paciente
a. experimentación de un cambio de estado
b. tema incremental ${ }^{3}$
c. afectación causal por otro participante
d. posición inmóvil con respecto al movimiento de otro participante
e. no existencia independiente del evento, o no existencia de ningún tipo

El modo en que los proto-papeles se implican en la estructura argumental viene determinado por el principio de selección argumental (Dowty, 1991: 576):

(4) En predicados con sujeto y objeto gramatical, el argumento para el cual el predicado implica el mayor número de propiedades de Proto-Agente será lexicalizado como el sujeto del predicado; el argumento que posea el mayor número de implicaciones de ProtoPaciente será lexicalizado como el objeto directo.

Con este enfoque se asume que todas las implicaciones léxicas son igualmente importantes y, en consecuencia, no son necesarias las relaciones de precedencia entre las propiedades. La teoría de los proto-papeles temáticos soluciona de este modo el problema de las jerarquías temáticas y, además, ha sido verificada empíricamente tanto en el plano psicológico (Kako, 2006) como recientemente en el ámbito la lingüística computacional (Reisinger y otros, 2015). Por ello, nos parece más adecuada también para afrontar la organización de los argumentos en el SN.

\subsection{Paralelismo entre estructuras verbales y nominales}

La hipótesis de correspondencia temática (Giorgi y Longobardi, 1991: 29) establece que los verbos y sus sustantivos correspondientes poseen la misma estructura temática y seleccio-

3 Este concepto hace referencia al homomorfismo entre el desarrollo del evento y el cambio progresivo en la naturaleza del objeto designado por el mismo (Dowty, 1991: 567). Por ejemplo, en El jardinero corta el césped, se puede deducir el aspecto del evento a partir del estado del césped, es decir, la situación del césped permite saber si el evento 'cortar el césped' se ha iniciado, está en proceso de desarrollo o ha terminado, por lo que el césped constituye un tema incremental. Por el contrario, el estado del jardinero no permite concluir nada sobre el evento 'cortar el césped'. 
nan como externo el mismo papel- $\theta$. Por sustantivos correspondientes se hace referencia a los nombres deverbales, clase central en el estudio del paralelismo entre SV y SN, ya que parecen heredar la estructura argumental de sus verbos de origen y asignar papeles temáticos verbales a sus complementos. Nuestra propuesta supone ampliar esta hipótesis de correspondencia temática para incluir también a los nombres de representación, que también pueden considerarse como sustantivos correspondientes por estar vinculados semánticamente a un predicado verbal.

En el plano sintáctico, la hipótesis del sintagma determinante o SD (Abney, 1987) inauguraba el paralelismo estructural entre las construcciones oracionales y las nominales. Así, se han distinguido categorías funcionales equivalentes y tres niveles paralelos en las proyecciones de verbos y sustantivos. En el entorno verbal existe un primer nivel léxico-temático, que contiene el SV y el Sv, un segundo nivel flexivo, con el sintagma flexión o SF, y un último nivel discursivo, con el sintagma complementante o SC. De manera paralela, en el entorno nominal el nivel léxico-temático contiene al SN y al Sn, el nivel flexivo contiene al sintagma concordancia o SConc, y el nivel discursivo contiene al SD4.

\subsection{Posesivización de argumentos}

En la oración existe un argumento jerárquicamente superior al resto: el argumento externo o sujeto. En el entorno nominal, el posesivo ha sido propuesto como argumento más prominente o "sujeto" del SN (Cinque, 1980) debido a tres motivos fundamentales: su posición estructural superior (situado arriba en el árbol sintáctico), su concordancia flexiva con el núcleo (en español, en género y número) y su autonomía referencial (proporciona referencia al SN que encabeza manteniendo la suya propia).

Precisamente es esta independencia semántica la que posibilita el proceso denominado posesivización: sustitución de un argumento genitivo posnominal por un posesivo prenominal (Giorgi y Longobardi, 1991: 66). Si el posesivo es el argumento más prominente o "sujeto" del SN, aquel argumento que sea conmutable por un posesivo podrá ser considerado igualmente como "sujeto" del SN. Esta prueba de reemplazo afecta a un solo sintagma preposicional (SP) en genitivo —es decir, encabezado por la preposición de-y se ajusta a unas jerarquías temáticas muy similares a las verbales: \{Poseedor > Agente > Experimentante >

4 Si bien han sido numerosas las propuestas de categorías funcionales en la bibliografía generativa, adoptamos la fuerza economizadora propia del programa minimista (Chomsky, 1995) y emplearemos las estrictamente necesarias, las cuales, por otro lado, han sido debida y ampliamente justificadas (véanse, entre otros, Rizzi, 1997; Platzack, 2001; Grohmann, 2003). 
Beneficiario/Fuente/Locativo > Tema\}(Escandell, 1995: 57)5. En función de esta escala, un agente como de María en (5a) podrá posesivizarse al ocupar el primer lugar (5b), mientras que la sustitución del tema del paisaje (5c) quedará bloqueada por estar el agente por delante de él.

(5) a. la descripción de María $_{\mathrm{AG}}$ del paisaje TEMA $_{\text {TE }}$

b. $s u_{\mathrm{AG}}$ descripción del paisaje $\mathrm{TEMA}_{\mathrm{T}}$

c. * ${ }^{S} U_{\text {TEMA }}$ descripción de María $_{A G}{ }^{6}$

Sin embargo, los problemas que se mencionaban arriba en relación con las escalas temáticas verbales se mantienen en el ámbito nominal. En la mayoría de trabajos sobre la estructura argumental del SN se suele operar con los dos papeles- $\theta$ "verbales" más prototípicos, esto es, el agente y el tema (véase, por ejemplo, Roca, 2012: 126), pese a que existen otros, como el experimentante o el beneficiario. Al agente y al tema se les ha dado, junto con el poseedor, un asiento estructural en los árboles sintácticos nominales correspondientes (Ticio, 2010: 25). A los demás, en cambio, no se les ha ofrecido espacio estructural en los árboles sintácticos nominales.

\section{La proyección de argumentos en el SN}

El modelo de estructura sintáctica para el SN carece de una propuesta integradora de todos los argumentos nominales posibles, ya que solo toma en consideración los papeles- $\theta$ de naturaleza verbal más prototípicos (agente y tema), además del poseedor, papel- $\theta$ genuinamente nominal. Dada la reciente verificación cognitiva y computacional de la teoría de los proto-papeles temáticos (Kako, 2006; Reisinger y otros, 2015), creemos pertinente proponer su aplicación - por primera vez hasta donde llega nuestro conocimiento- al entorno nominal del español.

\subsection{Proto-Agente y Proto-Paciente en el SN}

En virtud de la hipótesis de correspondencia temática (Giorgi y Longobardi, 1991: 29), la dicotomía de proto-papeles temáticos, el Proto-Agente y el Proto-Paciente, debería funcionar

5 Evidentemente, el paralelismo pleno entre la jerarquía temática verbal y la nominal queda roto por la presencia en esta última del poseedor, papel- $\theta$ estrictamente nominal.

6 Nótese que la agramaticalidad de esta construcción se debe exclusivamente a la asignación temática: el posesivo no puede ser el tema si hay un SP agentivo presente. En cambio, si se alteran los papeles asignados, esto es, si María es lo descrito, la secuencia sería gramatical: su AG $_{\text {descripción }}$ de María tema. 
también en los SSNN encabezados por un núcleo deverbal. Desde este enfoque, si partimos del principio de selección argumental (Dowty, 1991: 576) y lo aplicamos al ámbito nominal, será sujeto del SN y, por tanto, se podrá posesivizar, aquel argumento que contenga un mayor número de implicaciones de Proto-Agente. Consideremos el siguiente ejemplo:

(6) a. la construcción de los romanos del puente

b. Los romanos construyeron el puente.

El sustantivo deverbal construcción (6a), para el que podemos suponer una estructura verbal paralela (6b), posee dos argumentos saturados en forma de SP: de los romanos y del puente. De los dos, es el primero (de los romanos) el que contiene más propiedades de Proto-Agente: los romanos se implican volitivamente en la construcción del puente, son una entidad humana, dotada de conciencia y percepción, causan un cambio de estado, se mueven con respecto al objeto (el puente) y existen con independencia del proceso de construcción. En cambio, el segundo argumento (del puente) experimenta un cambio de estado (de no existir a existir), es el tema incremental, se ve afectado por los constructores, permanece inmóvil y su existencia depende de los romanos, por lo que cumple las características de Proto-Paciente. De este modo, se asignan los proto-papeles a cada argumento (7a). Será el Proto-Agente el que acepte la posesivización (7b), erigiéndose, pues, como sujeto del SN y bloqueando el ascenso al posesivo del Proto-Paciente (7c):

(7) a. la construcción de los romanos ${ }_{P-A G}$ del puente P.PAC $_{\text {Pac }}$

b. $\underline{\text { sup }}_{\mathrm{PAG}}$ construcción del puente $\mathrm{p}$ PAC

c. * *U $_{P-P A C}$ construcción de los romanos $P$-AG

Parece, pues, que la dicotomía \{Proto-Agente / Proto-Paciente\} opera en los sustantivos deverbales. Los ejemplos que siguen poseen núcleos deverbales y ponen de manifiesto que siguen operativas las mismas condiciones de posesivización que apuntábamos en la sección anterior; la única diferencia es la nueva jerarquía temática \{Proto-Agente > Proto-Paciente\}.

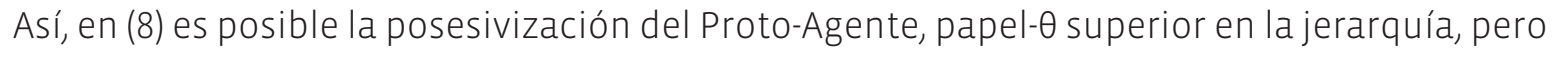
no la del Proto-Paciente, bloqueada por aquel, mientras que en (9) el Proto-Paciente sí puede posesivizarse por ser el único argumento presente:

(8) a. la traducción de Ana ${ }_{\text {P-Ag }}$ de la novela

b. $\underline{s u}_{P-A G}$ traducción de la novelap-PAC

c. * ${ }^{*} u_{P-P A C}$ traducción de Anap-AG

(9) a. la captura del enemigop-pac

b. Sup.PAC captura

Del mismo modo, las alteraciones en la realización sintáctica del Proto-Agente posibilitan la posesivización del Proto-Paciente. Así, en (10) el Proto-Agente va introducido por la locución 
prepositiva por parte de, con lo cual ha dejado de ser un argumento del núcleo y el SN tiene interpretación pasiva, mientras que en (11) el Proto-Agente no va en genitivo:

(10) a. la destrucción de las pruebas

b. $\underline{\text { Su}}_{\mathrm{P}-\mathrm{AG}}$ destrucción de las pruebas P.PAC $^{7}$

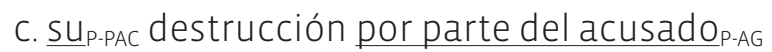

(11) a. la entrega del paquete ${ }_{\text {P.PAC }}$ al director ${ }_{\text {P-AG }}$

b. $\underline{\text { SU }}_{\mathrm{P} \text {-PAC }}$ entrega al director ${ }_{\mathrm{P}-\mathrm{AG}}$

c. ${ }^{*} \underline{S U}_{P-A G}$ entrega del paquete ${ }_{P-P A C}$

Cuando la preposición de un SP está regida por el núcleo, dicho SP argumental ya no puede posesivizarse, por lo que no puede ser el sujeto del SN, sino un complemento regido (Escandell, 1995: 60). En efecto, en (12) y en (13) la preposición de está regida por el núcleo, como muestran los verbos de origen (abusar de algo, depender de algo), de ahí que la posesivización no sea posible. En el resto de los casos - lo que muchos autores consideran un SN y no un SP - sí se permite la conmutación: en (14) el sustantivo grabación no selecciona la preposición de, ya que la estructura verbal de origen no la requería (grabar el disco)․․

(12) a. el abuso de pastillas

b. * $\underline{\text { su }}$ abuso

(13) a la dependencia del café

b. * su dependencia

7 ELSP introducido por la locución por parte de, al no estar en genitivo, no se puede posesivizar. Lo que se posesiviza aquí en realidad es el Proto-Agente genitivo de una estructura subyacente paralela:

(i) la destrucción del acusadop-ag de las pruebas P-PAC

8 Muchos autores (Giorgi y Longobardi, 1991: 23; Zagona, 2002: 146-149, entre otros) consideran que la preposición que introduce un argumento del nombre (di en italiano, de en español) es una preposición vacía (dummy preposition), ya que permite que la expresión referencial que introduce pueda ligar y mandar-c a elementos situados en el exterior del SP en que aparece. Según este análisis, estas preposiciones, herederas del caso genitivo latino, vacías de contenido léxico y no regidas por el núcleo (la traducción de Ana de la novela), no proyectarían un auténtico SP, sino que constituirían meras marcas de caso o indicadores de función del SN que introducen. Por tanto, solo las preposiciones regidas por el núcleo (el abuso de pastillas) proyectarían un auténtico SP. Otros autores, por el contrario, descartan esta ambivalencia de análisis: "La diferenciación entre una preposición de 'llena' y una preposición de ‘vacía' resulta ser, en alguna medida, cuestionable, particularmente si conlleva la anulación del estatus de SP del complemento del nombre en los Ilamados usos 'vacíos'” (Fernández Leborans, 2003: 64).

9 Téngase en cuenta que los únicos problemas de análisis podrían plantearse ante la rección de la preposición de, que es el "genitivo por defecto" en español, ya que cualquier otra preposición, al no asociarse al genitivo, impide por sí sola la posesivización. 
(14) a. la grabación del disco

b. su grabación

Por otro lado, pese a no ser deverbales en sentido estricto, los sustantivos de representación también pueden regirse por las implicaciones de Proto-Agente y Proto-Paciente, posiblemente debido a su vinculación con predicados de tipo verbal ${ }^{10}$. Además, permiten la presencia del papel de Poseedor, que ocupa siempre el primer lugar de la escala temática. Desde este nuevo enfoque de proto-papeles- $\theta$, el Poseedor siempre estará por delante del Proto-Agente y del Proto-Paciente, por lo que la jerarquía actualizada será \{Poseedor > Proto-Agente > Proto-Paciente\}:

(15) a. el retrato de Picasso ${ }_{P-A G}$ del coleccionista

b. $\underline{\text { supos }}_{\mathrm{pos}}$ retrato de Picassop-AG

c. * ${ }^{\text {SU }}$ P-AG retrato del coleccionistapos

(16) a. la foto de Dublín $\underline{p-p a c}_{\text {de Vanesapos }}$

b. $\underline{\text { supos }}_{\text {foto de Dublín }}$ f.PAC

c. * ${ }^{*} u_{p-p a C}$ foto de Vanesapos

Ambos núcleos nominales icónicos permiten la coexistencia de proto-papeles- $\theta$ "verbales" y un Poseedor, papel- $\theta$ puramente nominal (15a, 16a). Este, como elemento superior en la jerarquía, admite la posesivización en ambos casos (15b, 16b) y bloquea la del Proto-Agente (15c) y la del Proto-Paciente (16c). Conviene tener en cuenta, pues, que los nombres de representación se asimilan, en su comportamiento argumental, a los deverbales ${ }^{11}$.

10 No parece que se deriven de verbos, pero sí se vinculan semánticamente a un predicado — por ejemplo, retrato se asocia a retratar o pintar un retrato; foto, a fotografiar o hacer una foto-, de ahí que admitan papeles- $\theta$ verbales como el Proto-Agente y el Proto-Paciente. De hecho, puede haber vinculación morfológica, pero precisamente en el sentido inverso, es decir, el sustantivo da lugar al verbo denominal: retrato > retratar; fotografía > fotografiar.

11 También podrían ajustarse a la escala \{Poseedor > Proto-Agente > Proto-Paciente\} ciertos deverbales resultativos que designan el producto de un acontecimiento o proceso y parecen admitir un Poseedor.

(i) a. la adaptación del Quijotep.pac del niñopos

b. supos $_{\text {pdaptación del Quijotep.pac }}$

c. * ${ }^{\star}$ UP.PAC $_{\text {Pad }}$ adaptación del niñopos

(ii) a. la descripción de Unamunop-ag del profesor de literaturapos

b. ?supos descripción de Unamunop-Ag

c. * ${ }^{*} u_{P-A G}$ descripción del profesor de literaturapos

Si bien es cierto que en estos ejemplos se tiende a la lectura agentiva del segundo SP, no resultaría imposible su interpretación como Poseedor: en (ia) puede deducirse una lectura subyacente con Proto-Agente explícito ('el niño tiene la adaptación del Quijote que hizo Jordi Sierra i Fabra'), y en (iia), una paráfrasis del tipo ‘El profesor de literatura tiene una descripción de Salamanca escri- 
Parece, por tanto, solucionado el primer inconveniente de los numerosos papeles- $\theta$ y su ordenación, gracias a la adopción de la teoría de los proto-papeles temáticos. Así, se ha demostrado que la dicotomía \{Proto Agente / Proto-Paciente\} funciona en el caso de los SSNN encabezados por sustantivos deverbales y de representación. La cuestión que surge ahora es comprobar si también puede aplicarse al resto de SSNN con estructura argumental.

\subsection{Sustantivos con argumentos inherentes}

Hasta ahora se han empleado ejemplos de sustantivos que aceptaban los papeles- $\theta$ vinculados al ámbito verbal, debido fundamentalmente al hecho de que los estudios sobre la estructura argumental del SN en español (Demonte, 1985; Escandell, 1995; Picallo, 1999; Ticio, 2010; Peris, 2012; Fábregas, 2014, entre otros) parten de la organización de los complementos y funciones verbales, que se trasladan después al universo nominal. Se da por hecho que las propiedades temáticas de un nombre, cualesquiera que sean, se derivan de su verbo subyacente. Ahora bien, cabe plantearse si es posible que los principios de selección argumental sean exactamente los mismos para el verbo y para el nombre. Tomemos en consideración los siguientes ejemplos:

(17) a. el hermano de Sofía

b. la amiga de Pablo

c. la rama del árbol

d. la capital de Italia

En efecto, a estos nombres no se les puede suponer ninguna estructura verbal subyacente, ya que no denotan eventos o procesos. Se trata de otro grupo de sustantivos que tienen estructura argumental: Ios sustantivos relacionales, cuyo estudio parece haber quedado en un segundo plano en beneficio de las nominalizaciones ${ }^{12}$. Es evidente que no podemos hablar de propiedades de los proto-papeles temáticos "verbales" para sustantivos como los de (17): los predicados hermano, amiga, rama o capital no asignan a sus argumentos ninguna de las características semánticas de Proto-Agente o Proto-Paciente especificadas en (2) y (3) respec-

ta por Unamuno'. Así, mientras la posesivización del Proto-Paciente y del Proto-Agente provoca secuencias sin duda agramaticales (ic, iic), la del Poseedor da como resultado secuencias quizá dudosas, pero no totalmente inaceptables (ib, iib).

12 A pesar de que los sustantivos de representación suelen incluirse en el grupo de los relacionales, tienen un comportamiento distinto de estos porque en aquellos sí puede deducirse una estructura verbal subyacente, como ya se ha señalado (recuérdese la nota 10). Así, foto se vincula semánticamente a la actividad 'hacer una foto', aunque diacrónicamente fotografiar sea un derivado de foto. Por ello, desde el punto de la estructura argumental, se comportan igual que las nominalizaciones, a diferencia del resto de nombres relacionales, que podríamos considerar los relacionales "puros". 
tivamente. Sin embargo, cada uno de esos complementos preposicionales admite la posesivización, prueba de que se trata de argumentos, exactamente de los sujetos de esos SSNN (18), a diferencia de los sustantivos sin red temática, cuyos modificadores en forma de SP no se pueden posesivizar porque son adjuntos (19-22)

(18) a. su hermano

b. su amiga

c. su rama

d. su capital

(19) a. el vaso de cristal

b. * su vaso

(20) a. el autobús de las cinco

b. *su autobús

(21) a. una docena de huevos

b. * su docena

(22) a. la ciudad de Madrid

b. * su ciudad

La posesivización se convierte así en una prueba para determinar si un modificador nominal es argumental o no. Lo mismo ocurre en inglés, donde se puede aplicar la posesivización con genitivo sajón para discriminar argumentos (23) de adjuntos (24):

(23) a. the wife of Peter

b. Peter's wife

(24) a. the ring of gold

b. *the gold's ring

Resulta evidente, por tanto, que estos sustantivos tienen argumentos y que estos no pueden seleccionarse siguiendo las mismas implicaciones verbales de Proto-Agente y Proto-Paciente.

13 Los SSPP de posesión alienable o extrínseca constituyen un caso especial, ya que sí aceptan la posesivización: a pesar de no pertenecer a la red argumental del núcleo, se establece entre ambos una relación abstracta no fijada desde el léxico, esto es, una relación entre dos entidades $x$ e y cuyo contenido dependerá del contexto (Escandell, 1995: 41). Así, un SN como el ordenador de Ana admite diversas interpretaciones ('el ordenador que posee Ana', 'el que utiliza en el trabajo', 'el que quiere comprarse'...) en las que siempre están presentes los dos mismos argumentos (Ana y el ordenador), aunque nada en el contenido léxico de ordenador obligue a interpretar de Ana de una manera fijada. Por otro lado, en nombres con argumentos, el papel- $\theta$ de Poseedor aparece siempre como el prominente en las jerarquías temáticas, tal y como se ha señalado arriba. 
Para determinar qué principios motivan la selección argumental en estos SSNN será necesario profundizar más en la naturaleza de esta clase de predicados.

Disponemos de una prueba sintáctica eficaz, la posesivización, que no hace más que manifestar formalmente un fenómeno semántico: un nombre relacional establece, por su significado léxico, una relación diádica entre dos entidades $x$ e y; por el contrario, un nombre no relacional denota simplemente un conjunto monódico $x$ de entidades (Barker, 1995: 51). Véase el contraste entre hermano, sustantivo relacional, y vaso, sustantivo no relacional:

(25) a. [hermano] $=\{(x, y) x$ es hermano de $y\}$

b. $[$ vaso $]=\{x \mid x$ es un vaso $\}$

Asumiendo que la función gramatical de las construcciones posesivas es identificar uno de los participantes en la relación, es decir, señalar cuál es el "poseedor" y cuál es el "poseído" —entendidos estos conceptos en su sentido amplio (RAE y ASALE, 2009: 18.5a)—, solo podrán aceptar esta prueba los sintagmas de significado relacional. Se entiende por tales: en primer lugar, los encabezados por nombres de relación "puros" (como los nombres de parentesco o los que expresan una relación parte-todo); en segundo lugar, aquellos sintagmas en que se establecen relaciones de posesión alienable (aunque tal relación no esté fijada por el núcleo); en tercer lugar, las nominalizaciones deadjetivales, asimilables a una relación de "posesión" entre dos elementos: la cualidad y el "poseedor" de esa cualidad (por ejemplo, Juan "posee" inteligencia en la inteligencia de Juan ${ }^{14}$. Compárense los siguientes ejemplos de posesivización:

(26) a. el hermanox $\underline{\text { de Sofíay }}$

b. $\underline{\mathrm{su}}_{\mathrm{Y}} \underline{\text { hermanox }}$

(27) a. el vaso $\underline{\text { de }}$ cristal $x_{x 1}$

b. el vasox $\underline{\text { de plástico }} \underline{x 2}_{2}$

c. ${ }^{*} \underline{\mathrm{Su}}_{\mathrm{X} / \mathrm{X} 2} \underline{\mathrm{vaSO}} \mathrm{x}$

Como puede comprobarse, en (26) es necesario determinar que x es el elemento "poseído" e y el elemento "poseedor" en la relación que se establece entre ambos, pero en (27) solo hay un elemento $x$ con una propiedad $x 1$ (27a) o x2 (27b) que no se puede posesivizar $(27 c)^{15}$.

14 Quedan excluidos, lógicamente, los SSNN deverbales porque su estructura argumental es heredada de verbos y la posesivización no se ajusta a estos mismos criterios, sino a las propiedades de Proto-Agente y Proto-Paciente, como ya se ha señalado.

15 Nótese que no se pueden posesivizar estos SSPP porque son modificadores no argumentales de tipo circunstancial, pero sí se podría posesivizar un SP de posesión alienable que complementara al mismo núcleo:

(i) a. el vaso de Lucía

b. su vaso 
Por lo tanto, un sustantivo relacional consta de dos argumentos, que denominaremos respectivamente ARGUMENTO NUCLEAR, porque es siempre el N del SN y proyecta su referencia a la totalidad del sintagma, y ARGUMENTO COMPLEMENTANTE, que se realiza en forma de SP o posesivo:

(28) a. [el hermano de Sofía $\left._{k}\right]_{i}$

b. $\left[\underline{s u}_{k} \text { hermano }_{i}\right]_{i}$

El argumento nuclear (AgtN) del SD de (28) es hermano, núcleo N que determina la referencia (i) de todo el SD, mientras que el argumento complementante (AgtC), de referencia distinta ( $k$ ), es el SP de Sofía en (28a), posesivizado como su en (28b).

Algunas de estas relaciones semánticas poseen dos términos léxicos específicos, lo que permite que cualquiera de sus dos participantes desempeñe la función de AgtN. Dicho de otra manera, algunos sustantivos relacionales tienen un término inverso lexicalizado y otros no (Barker y Dowty, 1993: 51). Obsérvese el contraste entre las siguientes parejas de relaciones:

(29) a. $R_{1}=\{(x, y) \mid y$ es descendiente de $x\}$

b. el padre de su hijo

c. el hijo de su padre

(30) a. $R_{2}=\{(x, y) \mid y$ es una parte de $x$ tal que ninguna otra parte de $x$ está por encima de $y\}$

b. la cima de la montaña

c. la ¿? de la cima

En la relación de (29a) existen dos palabras para expresar las posibilidades de realización sintáctica de las entidades x e y: padre, si x ocupa la posición de AgtN (29b), e hijo, si y ocupa la posición de AgtN (29c). Sin embargo, en la relación de (30a) hay un término para cuando y es AgtN, cima (30b), pero no para cuando esa función está ejercida por x (30c). Así, la relación $\mathrm{R}_{1}$ tiene dos términos léxicos opuestos entre sí: padre e hijo, mientras que la relación $\mathrm{R}_{2}$ tiene uno de ellos, cima, pero carece del opuesto, ya que no hay ningún sustantivo relacional que exprese el término inverso exacto.

De este modo retomamos el problema de la selección argumental en los sustantivos de relación. Es preciso explicar las carencias sistemáticas en la distribución léxica de relaciones semánticas argumentales: en primer lugar, hay que averiguar por qué algunos sustantivos relacionales (por ejemplo, padre) tienen un término inverso lexicalizado (hijo) y otros no (cima); en segundo lugar, para este segundo grupo, conviene indagar las causas que motivan la lexicalización de argumentos en una dirección y no en la otra, esto es, por qué la relación $\mathrm{R}_{2}(30 \mathrm{a})$ tiene precisamente un término para cuando y es AgtN pero no para cuando lo es $x$. 


\subsection{Los proto-papeles temáticos nominales}

Dado que los principios verbales de selección argumental, con la dicotomía \{Proto-Agente / Proto-Paciente\}, no son adecuados para describir la selección argumental en los nombres relacionales, la solución pasa por adoptar un nuevo conjunto de proto-papeles temáticos válidos para el ámbito nominal, denominados Proto-Parte y Proto-Todo (Barker y Dowty, 1993: 55):

(31) Proto-Parte

a. Define o se sitúa en el límite del otro relatum.

b. Es una propiedad del otro relatum.

(32) Proto-Todo

a. Contiene totalmente al otro relatum como parte propia.

b. Es una entidad concreta.

Al igual que sucede con los proto-papeles verbales, las dos implicaciones de Proto-Parte y Proto-Todo son semánticamente independientes - aunque muchos argumentos de nombres relacionales posean ambas. En esta clase de nombres, el argumento para el cual el predicado denotado por el sustantivo implica el mayor número de propiedades de Proto-Todo será lexicalizado como el Argumento Complementante (AgtC); el argumento que posea el mayor número de implicaciones de Proto-Parte será lexicalizado como el Argumento Nuclear (AgtN). Veamos un ejemplo:

(33) a. la longitud ${ }_{\text {P.PAR }}$ de la mesa $a_{\text {P.TODO }}$

b. la manop.par de Sarap.TOdo

Como se observa en (33a), longitud es Proto-Parte (núcleo o AgtN) por ser una propiedad de la mesa y de la mesa es Proto-Todo (SP o AgtC) por ser una entidad concreta. De la misma manera, en (33b) mano es Proto-Parte al definir el límite de Sara y de Sara constituye el Proto-Todo por contener a su mano como parte propia.

Parece que ya se puede dar respuesta a las dos incógnitas planteadas arriba. Los sustantivos que tienen un término inverso lexicalizado se explican porque ninguno de los dos participantes de la relación tiene más propiedades de Proto-Parte o Proto-Todo que el otro: se trata de una relación simétrica en la que tanto $x$ como y pueden satisfacer propiedades de Proto-Parte y lexicalizarse como AgtN. Así, tanto padre como hijo pueden definir o contener al otro, además de ser una propiedad del otro o una entidad concreta, por lo que la lexicalización actúa en ambas direcciones y se generan los dos términos. En cambio, cima contiene una propiedad de Proto-Parte, ya que se sitúa en el límite del otro relatum (en el ejemplo de arriba, la montaña), el cual, a su vez, contiene a esta como parte propia, satisfaciendo así una 
propiedad de Proto-Todo y dando lugar a una relación asimétrica, en la que solo la entidad de Proto-Parte se lexicalizará como AgtN.

De acuerdo con las implicaciones de los proto-papeles temáticos nominales, los sustantivos que expresan relaciones de parentesco tendrán siempre un término inverso, ya que los dos miembros de la relación satisfacen las mismas propiedades de Proto-Parte y Proto-Todo (34), pero los que expresan relaciones asimétricas carecerán de su opuesto correspondiente, pues uno de los participantes contendrá al otro, implicación de Proto-Todo (35), o bien se situará en el otro o definirá su límite, implicación de Proto-Parte (36):

(34) a. padre / madre - hijo / hija

b. abuelo / abuela - nieto / nieta

c. marido - mujer

d. ancestro - descendiente

(35) a. pierna

b. rama

c. cabeza

d. capítulo

(36) a. esquina

b. superficie

c. frontera

d. perfil

A continuación se reformulan los nombres relacionales de (17) desde esta nueva perspectiva, con la correspondiente asignación de proto-papeles temáticos nominales y la variante con posesivización:

(37) a. el hermanop-par de Sofíap-Todo

b. $\underline{\text { SUP.TODO }}_{\text {hermano.PAR }}$

(38) a. Ia amigap-PAR de Pablop-TODo

b. Su P-TODO amigap-PAR

(39) a. la ramap.par del árbol $\left.\right|_{\text {P-TODo }}$

b. SUP-TODO $_{\text {ramaP-PAR }}$

(40) a. la capital p-par de Italia p-TODo

b. sup-TOdo $_{\text {capital }}$ P.PAR

Estas propiedades funcionan, por tanto, con los nombres de relación "puros", pero cabe preguntarse si es posible aplicarlo a todos los sustantivos con red argumental, es decir, conviene averiguar si el enfoque \{Proto-Parte / Proto-Todo\} puede trasladarse también a 
las nominalizaciones deverbales y deadjetivales, así como a los sustantivos icónicos o de representación ${ }^{16}$.

Consideremos primero los sustantivos procedentes de verbos. Antes hemos analizado los papeles- $\theta$ de nombres como construcción (6), traducción (8) o destrucción (10) desde el modelo de Proto-Agente y Proto-Paciente, y el resultado parecía satisfactorio: aquel argumento con más propiedades de Proto-Agente será el sujeto del SN y se podrá posesivizar. Repetimos aquí el ejemplo de (8) por conveniencia:

(41) a. la traducción de Anap-Ag de la novelap.-PAc

b. sup-AG traducción de la novela p.PAC $_{\text {P. }}$

c. * ${ }^{*} u_{P-P A C}$ traducción de Anap-AG

En primer lugar, en las nominalizaciones deverbales podemos hallar — si el verbo de origen es transitivo- más de un SP argumental seleccionado por el núcleo, mientras que en los relacionales solo aparece uno expreso, el $\mathrm{AgtC}^{17}$. Al no haber una relación entre solo dos entidades, en principio, no se podría aplicar el nuevo enfoque. En segundo lugar, para que este fuera válido, de Ana, argumento prominente y sujeto nominal, tendría que satisfacer más propiedades de Proto-Todo y lexicalizarse como AgtC, pero tanto 'Ana' como 'la novela' son entidades concretas (32b) y ninguna de ellas contiene (32a), define o se sitúa en el límite (31a) o es una propiedad de la otra (3בb). Por el contrario, 'Ana' tiene intención volitiva, percepción y causa un cambio de estado, cumpliendo las implicaciones de Proto-Agente (2). El mismo razonamiento puede aplicarse a los demás ejemplos: construcción, destrucción... En consecuencia, es preciso mantener los proto-papeles temáticos de Proto-Agente y Proto-Paciente para determinar la selección argumental en nombres deverbales.

Examinemos ahora el funcionamiento de los sustantivos de representación, para los que también habíamos asumido, con resultados positivos, los proto-papeles- $\theta$ verbales. Repetimos a continuación el ejemplo de (15) con la correspondiente asignación de Poseedor, ProtoAgente y Proto-Paciente:

(42) a. el retrato de Picasso $\underline{P-A g}$ del coleccionista

b. $\underline{\text { supos }}_{\mathrm{P}}$ retrato de Picasso

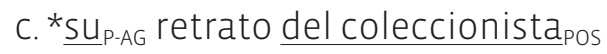

16 Recuérdese que estos nombres sí aceptaban las implicaciones verbales de Proto-Agente y ProtoPaciente, como se señaló arriba (\$3.1).

17 El otro argumento (AgtN), en realidad, es el que proyecta la referencia del núcleo. Así, en el hijo de Ana, el único argumento expreso es de Ana, porque el otro sería la entidad a la que hace referencia todo el sintagma proyectado por hijo (por ejemplo, Juan en El hijo de Ana es Juan). 
Al igual que los deverbales y a diferencia de los relacionales "puros", los nombres icónicos pueden tener más de un argumento posnominal realizado como SP. Sin embargo, para que se cumplieran las propiedades de los proto-papeles- $\theta$ nominales, solo uno podría materializarse como AgtC en forma de SP. Como en (42) encontramos dos SSPP argumentales, la asignación de implicaciones de Proto-Parte y Proto-Todo queda invalidada. Además, tanto el núcleo retrato como los SSPP de Picasso y del coleccionista constituyen entidades concretas (implicación de Proto-Todo), pero no definen (Proto-Parte) ni contienen (Proto-Todo) a ninguno de los otros, de modo que los tres deberían ser AgtC y aceptar la posesivización. En cambio, retrato se realiza como núcleo y solo es posesivizable del coleccionista, como muestra el contraste entre (42b) y (42c). Es preciso, pues, descartar la dicotomía \{Proto-Parte / Proto-Todo\} para los sustantivos de representación y conservar la jerarquía \{Poseedor > Proto-Agente > ProtoPaciente\} que proponíamos arriba.

Por último, analicemos las nominalizaciones deadjetivales o de cualidad, de cuyas semejanzas con los nombres relacionales ya hemos aportado algún que otro indicio (\$3.2):

(43) a. Ia amabilidad de Carmela

b. su amabilidad

(44) a. la rapidez del atleta

b. su rapidez

Todos los adjetivos hacen referencia a una entidad que constituye su argumento. En estos casos dicho argumento se manifiesta en el sustantivo derivado: de Carmela en (43) y del atleta en (44). En (43) sí podemos asumir que Carmela es una entidad concreta, satisfaciendo una característica de Proto-Todo, y que la amabilidad es una propiedad de Carmela, satisfaciendo una característica de Proto-Parte. En función de las implicaciones semánticas de estos protopapeles nominales, de Carmela se materializa como AgtC en forma de SP, con posibilidad de posesivización, y amabilidad se realiza como AgtN en forma de núcleo de todo el sintagma. Igualmente, la rapidez es una propiedad del atleta y este es una entidad concreta en (44), por lo que se aplica el mismo criterio. Por consiguiente, la asignación de los proto-papeles temáticos nominales es válida para los SSNN de núcleo deadjetival:

(45) a. la amabilidad ${ }_{\text {-PAR }}$ de Carmela

b. $\underline{\text { SUP-TODO }}_{\text {amabilidad }}$ P-PAR

(46) a. Ia rapidezp-par del atletap-TOdo

b. $\underline{\text { SUP-TODO }}_{\text {rapidez }}$ PAR

En resumen, la clase a la que pertenece el núcleo de un SN desempeña un papel esencial en el tipo de implicaciones semánticas por las que se rige su estructura argumental: unos se ajustan al esquema \{Proto-Agente / Proto-Paciente\}, otros siguen la dicotomía \{Proto-Parte / Proto-Todo\}. 


\subsection{La selección de argumentos en el SN español}

Una vez analizadas todas las clases de sustantivos con estructura argumental, estamos en condiciones de formular una versión revisada, actualizada y mejorada de los principios de selección argumental sobre la base de los proto-papeles temáticos (Dowty, 1991; Barker y Dowty, 1993). La organización de los argumentos en el SN español viene, por tanto, determinada por el que denominaremos Principio General de Selección Argumental Nominal:

(47) a. En Ios SSNN con estructura argumental heredada de verbos o vinculada a ellos (los de representación), el argumento genitivo correspondiente al Poseedor será lexicalizado como el sujeto de dicho SN; en ausencia de Poseedor, será sujeto el argumento genitivo para el cual el predicado implica el mayor número de propiedades de Proto-Agente; en ausencia de Proto-Agente, el argumento genitivo con mayor número de implicaciones de Proto-Paciente será lexicalizado como sujeto.

b. En los SSNN con estructura argumental heredada de adjetivos o de tipo relacional, el argumento genitivo para el cual el predicado implica el mayor número de propiedades de Proto-Todo será lexicalizado como el Argumento Complementante (AgtC); el argumento que posea el mayor número de implicaciones de Proto-Parte será lexicalizado como el Argumento Nuclear (AgtN).

De esta manera, la selección argumental en el SN se regirá por las directrices verbales cuando sus argumentos puedan asociarse a alguna de las implicaciones de la dicotomía \{Proto-Agente / Proto-Paciente\}, algo que sucede con los sustantivos deverbales, que denotan eventos o resultados, y con los nombres de representación, asociados a un predicado verbal. Sin embargo, se regirá por las directrices nominales cuando sus argumentos puedan asociarse a alguna de las implicaciones de la dicotomía \{Proto-Parte / Proto-Todo\}, circunstancia que se da con los sustantivos relacionales y con las nominalizaciones de cualidad.

Con el fin de incorporar los nuevos enfoques y planteamientos adoptados, necesitamos revisar el principio de posesivización (\$2.4). Tomando como punto de partida la teoría de los proto-papeles temáticos y considerando la doble posibilidad de selección argumental, formulamos la siguiente versión actualizada del Principio de Posesivización:

(48) En un SN dado, es conmutable por un posesivo:

a. el argumento genitivo en función de la jerarquía temática \{Poseedor > Proto-Agente > Proto-Paciente\}, siempre que la preposición no esté seleccionada léxicamente por el núcleo, si se trata de SSNN de núcleo deverbal o icónico.

b. el argumento genitivo que posea un mayor número de implicaciones de Proto-Todo, esto es, el AgtC, si se trata de SSNN de núcleo relacional o deadjetival. 
Solo un argumento aceptará la posesivización en función de una de las dos jerarquías — \{Poseedor > Proto-Agente > Proto-Paciente\} o \{Proto-Todo > Proto-Parte ${ }^{18}$ - , convirtiéndose así en el sujeto del SN. Los SSNN de (49) y (50) constituyen ejemplos de sustantivos deverbales y de representación, con sus correspondientes argumentos de naturaleza verbal:

(49) a. la descripción de Baroja $a_{P-A g}$ del paisaje ${ }_{P-P A C}$

b. $\underline{\text { sup }}_{\mathrm{P}-\mathrm{AG}}$ descripción del paisaje $\underline{P}_{\text {-PAC }}$

c. * ${ }^{\star} u_{p-p a c}$ descripción de Baroja $a_{p-A G}$

d. $\underline{s u}_{P . P A C}$ descripción por (parte de) BarojäP-AG

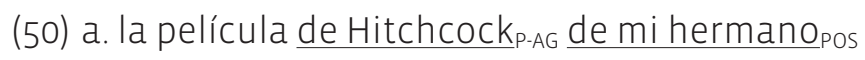

b. $\underline{s u}_{p o s}$ película de Hitchcock

c. ${ }^{*} \underline{S U}_{P-A G}$ película de mi hermanopos

En (49) operan los principios verbales de selección argumental porque el núcleo descripción es deverbal y los argumentos de Baroja y del paisaje se asocian más fácilmente a las propiedades de Proto-Agente y Proto-Paciente respectivamente: el argumento asociado a 'Baroja' es el Proto-Agente porque implica intención volitiva en el proceso de descripción, conciencia, etc., mientras que el asociado a ‘descripción' es el Proto-Paciente porque se ve afectado por otro participante, etc. (49a). Será posesivizable el Proto-Agente de Baroja al ser el superior en la jerarquía temática (49b), pero no el Proto-Paciente, pues es el último y hay un Proto-Agente expreso (49c), restricción que desaparece si el Proto-Agente deja de estar en genitivo (49d). El ejemplo de (50) pone de manifiesto que los sustantivos de representación se asimilan a los deverbales en lo que respecta a la selección argumental. El núcleo película está complementado por dos SSPP: un Proto-Agente, de Hitchcock, y un Poseedor, de mi hermano (50a). En función de la jerarquía temática \{Poseedor > Proto-Agente > Proto-Paciente\}, será gramatical la posesivización del Poseedor (50b), pero no la del Proto-Agente (50c).

Los SSNN de (51) y (52) constituyen ejemplos de sustantivos relacionales y deadjetivales con sus correspondientes argumentos de naturaleza nominal:

(51) a. el primop-PAR de Luis

b. $\underline{S U}_{\text {P-TODO }}$ Primop-PAR

(52) a. Ia inteligenciap-par de Susanap-Todo

b. Su $\underline{\text { P-TODO }}_{\text {inteligencia }}$-PAR

18 En realidad, la jerarquía \{Proto-Todo > Proto-Parte\} no es tal en un sentido estricto, ya que solo puede posesivizarse el Proto-Todo, el único en forma de SP, y nunca el Proto-Parte, siempre realizado como núcleo. Sin embargo, preferimos mantener, al menos nominalmente, la jerarquía \{Proto-Todo > Proto-Parte\} para guardar el paralelismo con la jerarquía \{Poseedor > Proto-Agente > Proto-Paciente\}. 
En (51) y en (52) se aplican los principios nominales de selección argumental. El núcleo primo en (51) establece una relación diádica entre dos entidades, una de las cuales, 'Luis', constituye una entidad concreta, satisfaciendo uno de los rasgos de Proto-Todo, y se realiza como AgtC en forma de SP (51a) o de posesivo (51b). El núcleo inteligencia en (52) implica una propiedad de la entidad concreta 'Susana': el primero será, pues, el AgtN Proto-Parte; el segundo, el AgtC Proto-Todo (52a), que aceptará la prueba de la posesivización (52b).

Estas jerarquías tienen un claro reflejo estructural y se explican por la posición que cada uno de los argumentos ocupa en el árbol sintáctico, de acuerdo con la la hipótesis de la uniformidad de la asignación temática o HUAT (Baker, 1988: 46). En (53a) se presenta la estructura de los SSNN encabezados por sustantivos deverbales y de representación; en (53b) se presenta la estructura de los SSNN encabezados por sustantivos relacionales y deadjetivales:

(53) a

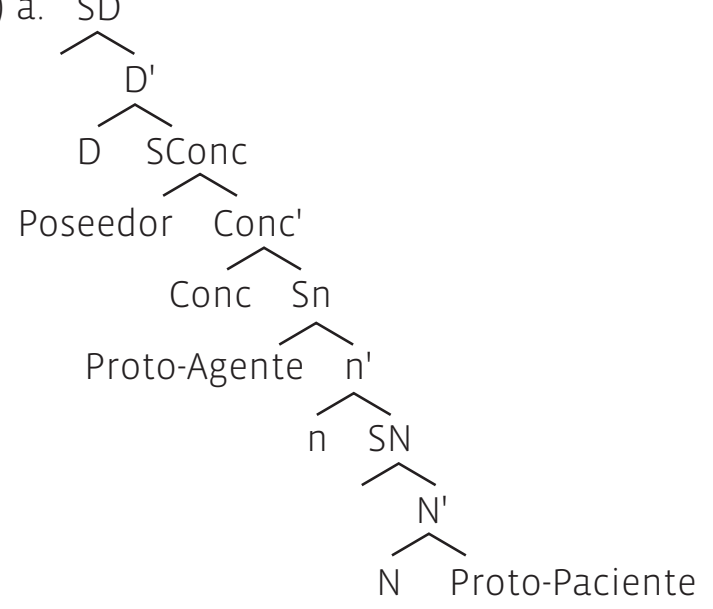

b. SD

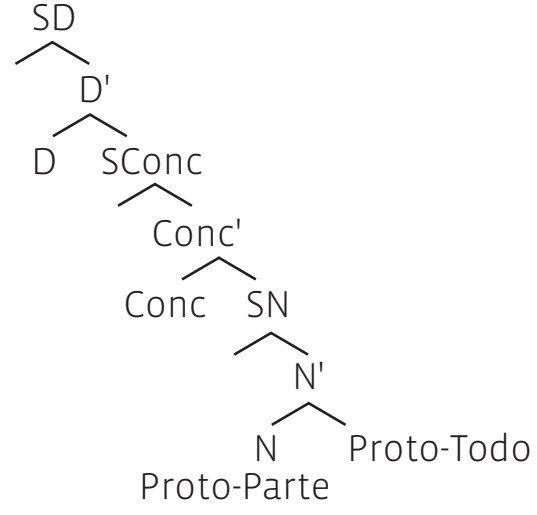

Así, en los SSNN de estructura argumental verbal (53a), que pueden tener hasta un máximo de tres argumentos, el Poseedor se ubica en la posición prominente en el especificador del sintagma concordancia; el Proto-Agente se sitúa por debajo, en el especificador de Sn, lugar de la agentividad, y el Proto-Paciente es el complemento del núcleo N. Por su parte, en los SSNN de estructura argumental nominal (53b), donde solo hay dos argumentos, el Proto-Parte es siempre el núcleo N, mientras que el Proto-Todo es siempre el complemento de $\mathrm{N}^{19}$.

19 Como puede observarse, en los SSNN con estructura argumental nominal no se proyecta la categoría funcional de Sn debido a la ausencia permanente de la noción de agentividad. En los SSNN con estructura argumental verbal en los que no hay Proto-Agente ni, por tanto, agentividad, tampoco se proyecta esta categoría. Las pruebas para la ausencia de un Sn pueden encontrarse en las diferencias de extracción entre los agentes introducidos por de y los introducidos por por (Ticio, 2010: 65). En el ámbito de la oración, Bošković (1997: 50) demuestra comportamientos similares para el Sv, el correlato oracional de Sn. 


\section{Conclusiones}

Este trabajo reformula los principios de selección argumental en el ámbito nominal español en función de la teoría de los proto-papeles temáticos y teniendo en cuenta la naturaleza específica de cada clase de SN con estructura argumental. La clasificación propuesta podría sintetizarse en el siguiente cuadro:

\section{CUADRO 1}

Tipos de estructura argumental en SSNN

\begin{tabular}{cccc}
$\begin{array}{c}\text { ESTRUCTURA } \\
\text { ARGUMENTAL }\end{array}$ & HEREDADA & INHERENTE & PROTO-PAPELES- $\boldsymbol{\theta}$ \\
Verbal & $\begin{array}{c}\text { Sustantivos } \\
\text { deverbales }\end{array}$ & $\begin{array}{c}\text { Sustantivos de } \\
\text { representación }\end{array}$ & $\begin{array}{c}\text { Proto-Agente } \\
\text { Proto-Paciente }\end{array}$ \\
\hline Nominal & $\begin{array}{c}\text { Sustantivos } \\
\text { deadjetivales }\end{array}$ & $\begin{array}{c}\text { Sustantivos } \\
\text { relacionales }\end{array}$ & $\begin{array}{c}\text { Proto-Todo } \\
\text { Proto-Parte }\end{array}$ \\
\hline
\end{tabular}

Así, el criterio distintivo fundamental para todos aquellos SSNN con red temática no es el carácter heredado o inherente de la estructura argumental, sino los principios de proyección de argumentos que desencadenan el proceso: los sustantivos deverbales y de representación se rigen por los mismos patrones que los verbos, con proto-papeles- $\theta$ verbales (Proto-Agente y Proto-Paciente), mientras que los nombres deadjetivales y relacionales se ajustan a un nuevo modelo nominal de proto-papeles- $\theta$ (Proto-Parte y Proto-Todo).

La idea subyacente a este análisis es que no es posible explicar la estructura argumental del SN basándose solo en el paralelismo con el SV, trasladando las pautas de organización de los argumentos verbales al universo nominal. Existen, en realidad, dos tipos de estructura argumental en el SN claramente diferenciadas, cada una con sus reglas particulares: la verbal —\{Proto-Agente / Proto-Paciente\}_y la nominal: \{Proto-Todo / Proto-Parte\}.

Nótese que con este modelo se mantiene la hipótesis de correspondencia temática (Giorgi y Longobardi, 1991: 29), que prevé que los verbos y sus sustantivos correspondientes poseen la misma estructura temática y seleccionan como externo el mismo papel- $\theta$. La única modificación consiste en considerar que esos sustantivos correspondientes no son solo los deverbales, sino también, por vinculación semántica, los de representación. Los sustantivos deadjetivales y relacionales, en cambio, quedan excluidos de esta hipótesis: su estructura argumental no guarda ninguna relación con los verbos, por lo que es preciso postular para ellos nuevos principios de selección argumental estrictamente nominales.

Nuestra propuesta de selección argumental para el SN en español ofrece una conceptualización novedosa y más precisa del paralelismo entre la legitimación argumental de nombres 
y verbos que se extiende más allá de los casos tradicionales. Pretendemos, así, proporcionar una caracterización teórica más abstracta de esta propiedad fundamental de las categorías léxicas introductoras de argumentos.

Este trabajo abre nuevas líneas de investigación en el ámbito de las ciencias cognitivas y la adquisición del lenguaje. Estamos de acuerdo con Marantz (2013: 165) cuando señala que la comprensión de la estructura argumental como constructo dentro de la sintaxis de una lengua con independencia de la información léxica encaja bien con el creciente interés cognitivo por la dicotomía entre lo memorizado y lo construido.

Pese a que la estructura argumental es uno de los componentes más importantes del desarrollo sintáctico, no se sabe mucho sobre cuándo y cómo aprenden los niños a unir los núcleos léxicos con sus correspondientes argumentos. Desde la obra inicial de Pinker (1989), algunos trabajos recientes han abordado la adquisición de la estructura argumental en verbos (Alishahi y Stevenson, 2008; Bavin y Stoll, 2013; Arunachalam, 2015; Peter y otros, 2015), pero aún no se ha llevado a cabo un estudio específico sobre la adquisición de la estructura argumental en sustantivos. En este sentido, sería interesante comprobar cómo y cuándo se adquieren los distintos tipos de sustantivos que poseen estructura argumental -los deverbales, los de representación, los deadjetivales y los relacionales - o si existen diferencias en la adquisición de SSNN de estructura argumental inherente (de representación y relacionales) o heredada (deverbales y deadjetivales). Asimismo, desde el ámbito de la neuropsicología cognitiva, podría analizarse el procesamiento de los nombres con estructura argumental a través de estudios de neuroimagen. De esta forma podría comprobarse, por ejemplo, si el paralelismo que hemos propuesto entre los sustantivos deverbales y de representación se refleja también en la manera en que ambos se procesan.

\section{Bibliografía citada}

Abney, Steven, 1987: The English Noun Phrase in its Sentential Aspect. Tesis doctoral, MIT.

Alexiadou, Artemis, Liliane Haegeman y Melita Stavrou, 2007: Noun Phrase in the Generative Perspective, Berlín: Mouton de Gruyter.

Alishahl, Afra, y Suzanne Stevenson 2008: "A computational model of early argument structure acquisition”, Cognitive Science 32, 789-834.

Arunachalam, Sudha, 2015: "Argument structure: Relationships between theory and acquisition" en Roberto Almeida y Christina Manoullidou (eds.): Cognitive Science Perspectives on Verb Representation and Processing, Nueva York: Springer, 259-280.

BARKER, Mark, 1988: Incorporation: A Theory of Grammatical Function Changing, Chicago: University of Chicago Press. 
BARKer, Chris, 1995: Possessive Descriptions, Stanford, California: CSLI Publications.

BARker, Chris, y David Dowty, 1993: "Non-verbal Thematic Proto-Roles" en Amy Schafer (ed.): Proceedings of NELS 22, Amherst, Massachusetts: University of Massachusetts GLSA, 49-62.

Bavin, Edith, y Sabine Stoll, 2013: The acquisition of ergativity, Amsterdam: John Benjamins.

Bošković, Željko, 1997: The Syntax of Nonfinite Complementation: An Economy Approach, Cambridge, Massachusetts: MIT Press.

Bosque, Ignacio, y Javier Gutiérrez-ReXach, 2009: Fundamentos de sintaxis formal, Madrid: Akal.

Bresnan, Joan, y Jonni Kanerva, 1989: "Locative Inversion in Chichewa: A Case Study of Factorization in Grammar", Linguistic Inquiry 20, 1-50.

Chomsky, Noam, 1970: "Remarks on Nominalization" en Roderick Jacobs y Peter Rosenbaum (eds.): Readings in English Transformational Grammar, Waltham, Massachusetts: Blaisdell, 184-221.

Chomsky, Noam, 1981: Lectures on Government and Binding, Dordrecht: Foris.

Chomsky, Noam, 1995: The Minimalist Program, Cambridge, Massachusetts: MIT Press.

Cinque, Guglielmo, 1980: "On Extraction from NP in Italian”, Journal of Italian Linguistics 5, 47-99.

Demonte, Violeta, 1985: "Papeles temáticos y sujeto sintáctico en el sintagma nominal”, Rivista di Grammatica generativa 9-10, 265-331.

DowTy, David, 1991: “Thematic proto-roles and argument selection”, Language 67, 547-619.

Escandell, Victoria, 1995: Los complementos del nombre, Madrid: Arco-Libros.

FABREGAS, Antonio, 2014: "Argument structure and morphologically underived nouns in Spanish and English", Lingua 141, 97-120.

Fernandez Leborans, María Jesús, 2003: Los sintagmas del español: el sintagma nominal, Madrid: Arco-Libros.

Giorgl, Alessandra, y Giuseppe Longobardi, 1991: The syntax of Noun Phrases, Cambridge: CUP.

Giustı, Giuliana, 2008: "Agreement and Concord in Nominal Expressions" en Cécile de CAT y Katherine Demuth (eds.): The Bantu-Romance Connection. A comparative investigation of verbal agreement, DPs, and information structure, Amsterdam: John Benjamins, 201-237.

Grimshaw, Jane, 1990: Argument Structure, Cambridge, Massachusetts: MIT Press. 
Grohmann, Kleanthes, 2003: Prolific Domains: On the anti-locality of movement dependencies, Amsterdam: John Benjamins.

Gutiérrez Bravo, Rodrigo, 2007: "Prominence Scales and Unmarked Word Order in Spanish", Natural Language and Linguistic Theory 25, 235-271.

JACKENDOFF, Ray, 1972: Semantic interpretation in generative grammar, Cambridge, Massachusetts: MIT Press.

KAKo, Edward, 2006: "Thematic role properties of subjects and objects”, Cognition 101, 1-42.

LongOBARDI, Giuseppe, 2003: "The Structure of DPs: some principles, parameters and problems" en Mark Baltin y Chris Coluıns (eds.): The Handbook of Contemporary Syntactic Theory, Oxford: Blackwell, 562-603.

Marantz, Alec, 2013: "Verbal argument structure: Events and participants”, Lingua 130, 152-168.

PerIs, Aina, 2012: Nominalizaciones deverbales: denotación y estructura argumental. Tesis doctoral, Universitat de Barcelona.

Pesetsky, David, 1995: Zero syntax: Experiencer and cascades, Cambridge, Massachusetts: MIT Press.

Peter, Michelle, Franklin Chang, Julian Pine, Ryan Blything y Caroline Rowland, 2015: "When and how do children develop knowledge of verb argument structure? Evidence from verb bias effects in a structural priming task", Journal of Memory and Language 81, 1-15.

PICALlo, Carme, 1999: "La estructura del sintagma nominal: Ias nominalizaciones y otros sustantivos con complementos argumentales” en Ignacio Bosque y Violeta Demonte (dirs.): Gramática descriptiva de la lengua española, Madrid: Espasa-Calpe, 363-393.

PInker, Steven, 1989: Learnability and Cognition: The Acquisition of Argument Structure, Cambridge, Massachusetts: MIT Press.

Platzack, Christer, 2001: "Multiples Interfaces" en Emile van der Zee y Urpo Nikanne (eds.): Cognitive Interfaces. Constraints on Linking Cognitive Information, Oxford: OUP, 21-53.

Real Academia Española (RAE) y Asociacion de Academias de la Lengua Española (ASALE), 2009: Nueva gramática de la lengua española, Madrid: Espasa-Calpe.

Recıo, Álvaro, 2015: La estructura argumental del sintagma nominal en español. Tesis doctoral, Universidad de Salamanca.

Reisinger, Drew, Rachel Rudinger, Rachel Ferraro, Craig Harman, Kyle Rawlins y Benjamin van Durme, 2015: "Semantic proto-roles", Transactions of the Association for Computational Linguistics 3, 475-488. 
ReULAND, Eric, 2011: "What's nominal in nominalizations?”, Lingua 121, 1283-1296.

Rigau, Gemma, 1999: "La estructura del sintagma nominal: Ios modificadores del nombre" en Ignacio Bosque y Violeta Demonte (dirs.): Gramática descriptiva de la lengua española, Madrid: Espasa-Calpe, 311-362.

RIzzı, Luigi, 1997: “The Fine Structure of the Left Periphery” en Liliane HaEgeman (ed.): Elements of Grammar. Handbook of Generative Syntax, Dordrecht: Springer, 281-337.

Roca, Francesc 2012: "Procesos de movimiento internos a las construcciones nominales" en José M. ${ }^{a}$ Brucart y Ángel Gallego (eds.): El movimiento de constituyentes, Madrid: Visor Libros, 125-149.

SILonı, Tal, 1997: Noun Phrases and Nominalizations: the Syntax of DPS, Dordrecht: Springer.

Ticio, Emma, 2005: "Locality and Anti-locality in Spanish DPs", Syntax 8.3, 229-286.

Ticıo, Emma, 2010: Locality Domains in the Spanish Determiner Phrase, Dordrecht: Springer.

ZaGONA, Karen, 2002: The Syntax of Spanish, Cambridge, CUP. 